\title{
Thermal Imaging Fire Detection Algorithm with Minimal False Detection
}

\author{
Soo-Young Jeong ${ }^{1}$ and Won-Ho Kim ${ }^{1 *}$ \\ ${ }^{1}$ Department of Electrical, Electronic and Control Engineering, Kongju National University \\ Cheonan, Republic of Korea \\ [e-mail: whkim@kongju.ac.kr] \\ *Corresponding author: Won-Ho Kim
}

Received November 17, 2019; revised February 18, 2020; accepted March 15, 2020; published May 31, 2020

\begin{abstract}
This paper presents a fire detection algorithm with a minimal false detection rate, intended for a thermal imaging surveillance environment, whose properties vary depending on temporal conditions of day or night and environmental changes. This algorithm was designed to minimize the false detection alarm rate while ensuring a high detection rate, as required in fire detection applications. It was necessary to reduce false fire detections due to non-flame elements occurring when existing fixed threshold-based fire detection methods were applied. To this end, adaptive flame thresholds that varied depending on the characteristics of input images, as well as the center of gravity of the heat-source and hot-source regions, were analyzed in an attempt to minimize such non-flame elements in the phase of selecting flame candidate blocks. Also, to remove any false detection elements caused by camera shaking, one of the most frequently raised issues at outdoor sites, preliminary decision thresholds were adaptively set to the motion pixel ratio of input images to maximize the accuracy of the preliminary decision. Finally, in addition to the preliminary decision results, the texture correlation and intensity of the flame candidate blocks were averaged for a specific period of time and tested for their conformity with the fire decision conditions before making the final decision. To verify the fire detection performance of the proposed algorithm, a total of ten test videos were subjected to computer simulation. As a result, the fire detection accuracy of the proposed algorithm was determined to be $94.24 \%$, with minimum false detection, demonstrating its improved performance and practicality compared to previous fixed threshold-based algorithms.
\end{abstract}

Keywords: video surveillance system, IR image processing, thermal image processing, fire detection, digital signal processing 


\section{Introduction}

A forest fire or general fire is a disaster that harms the natural ecosystem and inflicts significant human and physical damage on the national economy and society. To prevent such disasters, a great deal of research has been conducted on early detection of forest fires or outdoor and indoor fires. Meanwhile, existing fire or forest fire detection methods have primarily used detection systems based on physical sensors that can detect smoke or heat. However, because the detection is made only when the flame or smoke reaches the physical sensors, such methods involve a delay in detecting flame or smoke when a fire occurs. Also, due to the limited range of fire detection, these methods are unsuitable for broad outdoor areas or forest fires. To overcome these problems, there has been active researches carried out to develop image processing-based smart fire surveillance solutions using cameras capable of monitoring wide areas. Furthermore, these solutions have been extensively applied in practice to the fire surveillance field. Two types of systems are commonly used for video surveillance. One is a color camera system [2]-[7],[12],[13],[16], and the other is an infrared(IR) camera system [1],[8]-[11],[17].

There are many existing algorithms to detect fire using color or infrared images. First, the color image based algorithms are as follows. In the paper [2], the algorithm comprises pixel-based fire motion and color analysis, with temporal analysis using a wavelet transform and spatial analysis using energy calculations of the fire area. In the paper [3], the authors propose a pixel-based fire color modeling that was developed in CIE-Lab color space, and fire motion analysis is used to detect fire events. In the paper [4], pixel-based fire motion is detected by background subtraction, edge detection, flame height, and width analysis, and color analysis methods are used for fire detection. In the paper [5], pixel-based frame difference calculations are used to extract the moving fire area, and color analysis and optical flow computation of dynamic features extraction methods are applied for detecting fire. In the paper [6], fire regions are detected using pixel-based potential fire region extraction processing with a spectral and spatial model, and temporal property calculations using Fourier coefficients are used to detect fire regions. In the paper [7], a pixel-based modified histogram back-projection algorithm is used to detect flame regions. In the paper [12], authors present pixel-based color analysis using a Gaussian smoothed color histogram, fire motion analysis based on temporal variations in pixels, and erosion and region growing are performed to detect fire. In the paper [13], the authors propose a forest-fire monitoring system based on field information, infrared and visual image processing. This system is based on forest fire properties such as the fire front, flame height, flame inclination angle, fire base width, a three dimensional perception model, sensor fusion techniques involving telemetry sensors, and GPS. In the paper [16], the authors propose a fire detection algorithm which used image enhancement technique, $\mathrm{RGB}$ and $\mathrm{YC}_{\mathrm{b}} \mathrm{C}_{\mathrm{r}}$ color models with given conditions to separate fire pixel from background and isolates luminance from chrominance contrasted from original image to detect fire.

Secondly, IR image based algorithms are as follows. In the paper [1], fire alarm is decided solely based on the fixed threshold-based candidate block selection and the average degree of correlation measured from input images to check flame motion. In the paper [8], the different behaviors of medium (3-5 $\mu \mathrm{m})$ and long $(8-12 \mu \mathrm{m})$ IR spectral regions are used to calculate the two fire-index using two IR cameras working in the medium and thermal IR spectral windows, which are used to identify fires. In the paper [9], several software components based on 
threshold, oscillation detection, matching of visual and IR images, memory, meteorologic information, motion, size, shape, solar conditions, and location are used to implement personal computer-based fire alarm system. In the paper [10], The proposed forest fire detection system combines computer vision tools, neural networks, and expert fuzzy rules to detect forest fires in open areas. In the paper [11], authors propose a forest-fire detection system based on images obtained from IR cameras. The algorithm utilize noise predictor to get better probability of detection. The noise predictor will compute the noise level of the cell under test. Then, this estimated level may be subtracted from the pixel under test, thus improving SNR and fire detection probability. In the paper [17], $940 \mathrm{~nm}$ narrow-band filter is applied to the camera to obtain an infrared image. Then, flame threshold and roundness of contour are analyzed to decide fire.

These image processing-based smart fire surveillance systems mainly use color cameras, along with specific fire detection algorithms that analyze the color or motion of flame for fire detection. In these color image processing-based smart fire surveillance systems, flame colors are analyzed by examining the multiple color elements that constitute the standard color space, such as RGB, $\mathrm{YC}_{b} \mathrm{C}_{\mathrm{r}}$, and $\mathrm{HSV}$, and flame motion information. Thus, a great deal of data needs to be processed, requiring a significant amount of processing time. Also, using CCD or CMOS color cameras, it is difficult to obtain images or avoid image quality degradation at night, without a light source, or in unfavorable weather conditions. This inevitably leads to an inability to perform fire surveillance or to degrade detection performance [2]-[7],[12],[13],[16].

To overcome these constraints of limited operating conditions, extensive data processing, and long processing time, infrared thermal imaging-based smart fire surveillance methods have been introduced as alternatives because these methods are immune to the given surveillance environment and simple to use when performing real-time processing, given the simplicity of the image data used. An infrared camera is a device that forms an image by detecting the radiant energy emitted by the vibrating and rotating atoms and molecules contained in the subject. Here, it is worth noting that every object that is above absolute zero temperature emits radiant energy. Thus, this type of system is capable of taking images whenever desired, day or night, and regardless of weather conditions. Also, its images express the thermal energy of the subject using intensity variations, and therefore the amount of data to be processed is less when compared to color images [1],[8]-[11],[17].

The infrared wavelength band of an infrared camera used for image-based surveillance includes the mid-infrared spectral range of 3-5 $\mu \mathrm{m}$ and far-infrared range of $8-12 \mu \mathrm{m}$. Infrared radiation is electromagnetic radiation with wavelengths longer than those of visible light and wavelengths shorter than communications wavelengths, i.e., 0.75 to $1,000 \mu \mathrm{m}$. To be more specific, near-infrared is defined as radiation with a wavelength of $0.75-3 \mu \mathrm{m}$; mid-infrared is $3-6 \mu \mathrm{m}$; far-infrared is $6-15 \mu \mathrm{m}$; and extreme infrared is $15-1,000 \mu \mathrm{m}$. Given the wavelength region of the energy emitted from surveillance targets, the detection characteristics of detectors, and the atmospheric transmission properties, in practice only part of this infrared radiation can be used, i.e., near-infrared with a wavelength near $1 \mu \mathrm{m}$, mid-infrared with a wavelength of 3-5 $\mu \mathrm{m}$, and far-infrared with a wavelength of $8-12 \mu \mathrm{m}$. There are two types of IR cameras, cooled type and non-cooled type. The non-cooled type of camera has being extensively applied for civilian surveillance applications. This non-cooled type infrared camera does not need a cooler, and thus it is easier to achieve lightweight design and low cost compared to when cooled type infrared cameras are applied; however, this camera's long-distance surveillance capability is inferior to that of cooled type infrared cameras. 
In existing infrared thermal imaging-based flame detection methods, flame candidate regions are extracted using fixed threshold values, and flame motion is analyzed for fire determination. Accordingly, the procedure for determining flame candidate regions from thermal images significantly affects the accuracy of the flame detection results and the amount of post-processing computation. Given that potential flame regions are expressed with high intensity thermal images, it is possible to detect flame regions simply by using a fixed threshold method. However, there exist elements that cause false detection, such as the steel structures, vehicle engines, roof of building and artificial lighting heated by reflected sunlight or surrounding environments. Also, the contrast and intensity of thermal images may vary depending on the characteristics of the camera used or the environments where it is installed. Therefore, to reduce false detection, thresholds for extracting flame regions must be adaptively set to the environment from which target images have been obtained.

The present study is composed as follows. In Chapter 2, a thermal imaging fire surveillance algorithm to implement a smart fire surveillance system with a minimal false detection rate is described. This algorithm consists of three phases, as follows: selection of flame candidate blocks using flame thresholds adaptive to temporal conditions such as day or night, and weather conditions, along with center-of-gravity analysis; analysis of temporal texture correlation of flame candidate blocks; and statistical preliminary and final fire decisions. In Chapter 3, computer simulation results of test images using the proposed algorithm are presented. Finally, in Chapter 4, the conclusions of the present study are described.

\section{Thermal Imaging Fire Detection Algorithm}

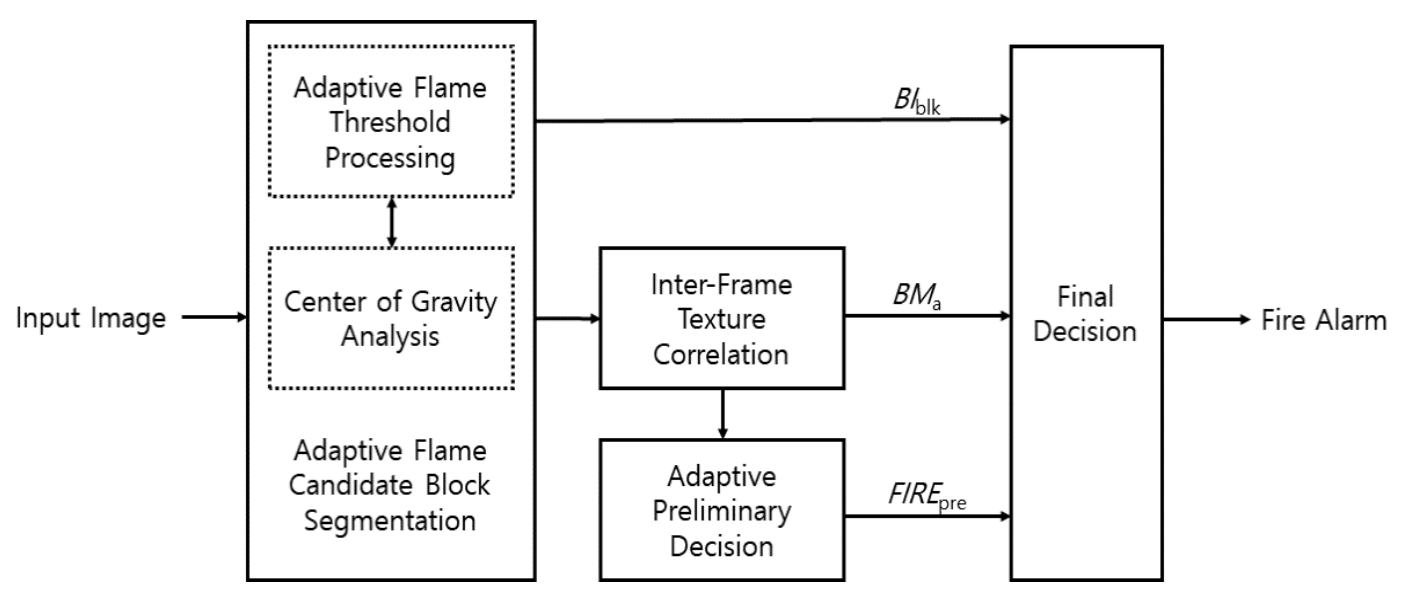

Fig. 1. Functional diagram of fire detection algorithm

A structural diagram of the thermal imaging fire detection algorithm proposed in the present study is shown in Fig. 1. The algorithm consists of selection of adaptive flame candidate blocks; analysis of temporal texture correlation of flame candidate blocks; and preliminary and final fire decisions. Input images were divided into $4 \times 4$ image blocks, and all operations were processed on a block-by-block basis. 


\subsection{Selection of Adaptive Flame Candidate Blocks}

To minimize the occurrence of non-flame elements in the early stage, the selection process of adaptive flame candidate blocks is divided into two phases of processing adaptive flame thresholds and analyzing the center of gravity, and these two phases are continuously conducted. As a first step, adaptive flame threshold processing is carried out, as follows.

\section{i) Adaptive Flame Threshold Processing}

Heat-source regions found in infrared thermal images are composed not only of potential flame regions but also elements that may cause false detection, such as reflected sunlight, vehicle engines, and other heating objects, as well as artificial lighting, such as traffic lights on the street. Existing fixed threshold-based selection methods of flame candidate blocks, however, have a limitation in that the intensity or contrast of input images may vary depending on the given surveillance environment, such as temporal conditions of day or night, and weather and local conditions, thereby causing false detection. To overcome this problem, a method of adaptively setting flame thresholds using the average intensity and standard deviation of input images was selectively applied. Given that the flame regions that need to be extracted have a higher intensity than the background, flame thresholds are adaptively set to the average intensity level of an input image using average intensity and standard deviation. Most pixels in the background have similar intensity levels close to the average value, while those in flame regions have very high-intensity levels due to their high thermal energy. Given these conditions, flame candidate blocks can be separated by setting a threshold larger than the sum of the average intensity and the standard deviation.

This method, however, causes false detection when obtained images are dark, as in tunnels, underground spaces, or night surveillance environments without light sources, because non-flame elements, such as moving people and vehicles, are often mistaken for flame candidate regions. This is because in such cases the overall temperature of the image appears low, and thus the average intensity of the image becomes close to zero, while the standard deviation is also small due to the relatively large number of background pixels. As a result, false detections may occur, where people or vehicles are mistaken for flame regions. To overcome all the problems mentioned above, a new selection method of flame candidate blocks was proposed that uses an adaptive threshold that reflects the maximum pixel value of an input image in addition to the average intensity and the standard deviation. In thermal images, the pixels contained in flame regions always tend to have the maximum intensity level, and thus adaptive thresholds that reflect this nature may ensure that flame candidate blocks can be more accurately separated in various surveillance environments than is possible with existing methods.

The proposed method of setting the adaptive thresholds is as follows. Here, two types of thresholds are used, the heat-source threshold $T H_{\text {heat }}$ and the hot-source threshold $T H_{\text {hot }}$, which separates hot-source regions. First, the heat-source threshold $T H_{\text {heat }}$ is defined as shown in Eq. 1. In the equation, $I_{\text {avg }}$ is the average intensity of an input image, and $I_{s t d}$ is its standard deviation. The standard deviation is multiplied by two to separate heat-source regions with higher intensity than the background region.

$$
T H_{\text {heat }}=I_{\text {avg }}+2 I_{\text {std }}
$$


The hot-source threshold $T H_{\text {hot }}$ is defined based on the maximum intensity of an input image, as shown in Eq. 2. $I_{\text {lmax }}$ is a value that is used for exception handling when the maximum intensity of the flame regions is lower. Simply put, when the maximum intensity of the input image is lower than $I_{\operatorname{lmax}}, I_{\max }$ is replaced by $I_{Y \max }$, the maximum intensity that can be achieved in the input image. $I_{l \max }$ was experimentally optimized to 179 , and $I_{Y \max }$, which represents the maximum intensity of the Y channel of the YCbCr color model, was set to 235. The $T H_{\text {heat }}$ and $T H_{\text {hot }}$ values determined above were reflected in determining the average intensity of image blocks and, as a result, heat-source and hot-source binary images were created. These two binary images were used later in the gravity center analysis of flame candidate regions.

$$
T H_{\text {hot }}=\left\{\begin{array}{l}
0.5\left(I_{\text {avg }}+3 I_{\text {std }}\right)+0.5 I_{Y \max }: \text { if } I_{\max }<I_{\text {lmax }} \\
0.5\left(I_{\text {avg }}+3 I_{\text {std }}\right)+0.5 I_{\max }: \text { otherwise }
\end{array}\right.
$$

\section{ii) Center-of-gravity Analysis}

A center-of-gravity analysis of the flame candidate regions extracted based on adaptive flame thresholds is intended to use heat-source and hot-source binary images to rule out non-flame elements with high intensity, thereby minimizing the occurrence of false fire detections. When these two binary images indicate that the center of gravity of the heat-source region and that of the hot-source region coincide, the corresponding region is classified as a flame region. In contrast, when they differ, the region is classified as a non-flame region. The rationale behind this classification is that, in actual flame regions, the center of gravity of a heat-source region tends to coincide with that of a hot-source region. In non-flame objects, such as moving vehicles and excavator engines, however, each center of gravity is differently located. Fig. 2 and Fig. 3 show that, in actual flame regions, the center of gravity of the heat-source region is almost identical to that of the hot-source region. In contrast, in the excavator engine, as a non-flame object, the centers of gravity do not coincide.
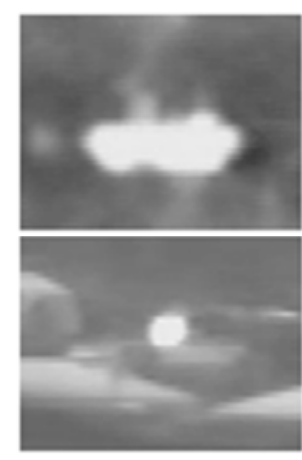

(Thermal Image)

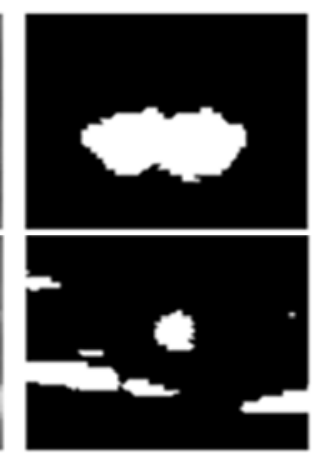

(Heat-source region)

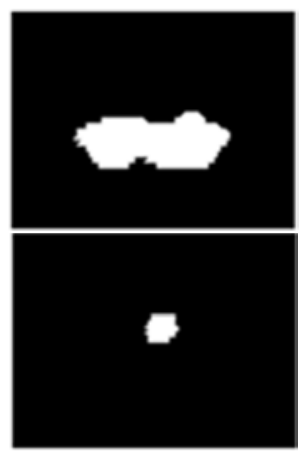

(Hot-source region)

Fig. 2. Comparison between heat-source regions and hot-source regions in fire image

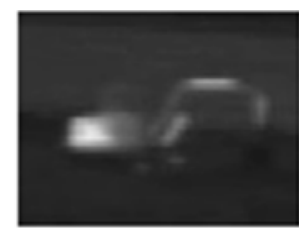

(Thermal Image)

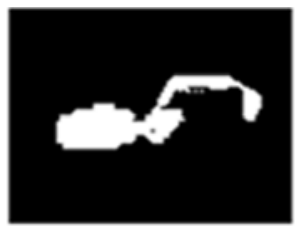

(Heat-source region)

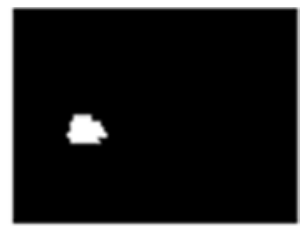

(Hot-source region) 
Fig. 3. Comparison between heat-source regions and hot-source regions in non-fire image

The coordinates of the heat-source and hot-source regions $\bar{x}$ and $\bar{y}$ can be estimated using Eq. 3. Here, $\mathrm{I}(\mathrm{x}, \mathrm{y})$ is the binary image coordinates, and $M_{00}$ is the number of pixels that constitute the flame region. Also, $M_{10}$ and $M_{01}$ are the sums of the x-coordinates and the $\mathrm{y}$-coordinates of the pixels that constitute the flame region, respectively.

$$
\bar{x}=\frac{M_{10}}{M_{00}}, \bar{y}=\frac{M_{01}}{M_{00}}, M_{i j}=\sum_{x} \sum_{y} x^{i} y^{j} I(x, y)
$$

The centers of gravity of the heat-source region and the hot-source region were calculated using Eq. 3, and the distance between each center of gravity DCOG was estimated using Eq. 4. Here, $\bar{x}_{\text {Heat }}$ and $\bar{y}_{\text {Heat }}$ are the coordinates of the heat-source region, while $\bar{x}_{H o t}$ and $\bar{y}_{H o t}$ are the coordinates of the hot-source region.

$$
\mathrm{DCOG}=\sqrt{\left(\bar{x}_{\text {Heat }}-\bar{x}_{\text {Hot }}\right)^{2}+\left(\bar{y}_{\text {Heat }}-\bar{y}_{\text {Hot }}\right)^{2}}
$$

When the distance between the centers of gravity is smaller than the center-of-gravity threshold $\mathrm{TH}_{d c o g}$, the concerned region is classified as a flame region. In contrast, when it is larger than the threshold, or there are no hot-source region pixels available, the region is classified as a non-flame region. The center-of-gravity threshold $\mathrm{TH}_{d \operatorname{cog}}$ was adaptively set to the size and shape of the flame regions, which were differently represented depending on the shooting position, e.g., from far or short distances. To this end, it was reflected that, in actual flame regions, the center of gravity of the heat-source region tended to be located within a specific radius of the center of gravity of the corresponding hot-source region. Accordingly, the threshold was adaptively set to ensure that any region could be classified as a flame region when the center of gravity of its hot-source region was located within a distance corresponding to the number of pixels defined from the center of gravity of its heat-source region. The estimation was performed as shown below.

1) The minimum and maximum values of the $\mathrm{x}$-axis and $\mathrm{y}$-axis for the hot-source region, i.e., $x_{\min }, x_{\max }, y_{\min }$ and $y_{\max }$ are determined.

2) Based on the minimum and maximum coordinates obtained, the reference distance $D C O G_{r e f}$ is calculated using Eq. 5. It is diagonal distance of virtual rectangular box surrounding hot-source region.

$$
\operatorname{DCOG}_{\text {ref }}=\sqrt{\left(x_{\max }-x_{\operatorname{mim}}\right)^{2}+\left(y_{\max }-y_{\min }\right)^{2}}
$$

3) The adaptive center-of-gravity threshold $T H_{d c o g}$ is estimated using Eq. 6. Here, $\alpha$ is the weighted value of the center-of-gravity threshold, and its optimal value was experimentally determined to be 0.1 . A small alpha means that the two centers of gravity are in about the same position. As the alpha value increases more than 0.1 , false alarm increases.

$$
T H_{\text {dcog }}=\alpha D C O G_{\text {ref }}
$$




\subsection{Analysis of Temporal Texture Correlation}

A temporal texture correlation analysis of flame candidate blocks is a method that analyzes their dynamic characteristics, such as the spread and motion of flame. Such texture change is measured using the correlation and average intensity of the candidate blocks contained in adjacent images. Here, the degree of correlation between each candidate block is estimated using a mean absolute difference method, which requires a small amount of computation [1]. In the case of false detection elements, such as objects reflected by sunlight, the degree of correlation is found to be high because these elements do not move over time. In contrast, actual flame regions exhibit a low degree of correlation due to the spread of flame and wind-driven flame motions. To implement this correlation analysis, candidate blocks and expanded blocks were defined, and the correlation between the flame candidate blocks (red square symbols) and expanded blocks (green square symbols) was indicated in Fig. 4.

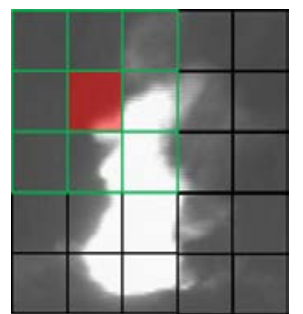

Fig. 4. Flame candidate blocks and expanded blocks

The degree of correlation for the candidate block located at the center $\left(B M_{a}\right)$ and for the expanded blocks $\left(B M_{b}\right)$ can be defined as shown in Eq. 7 and Eq. 8, respectively. Here, $\mathrm{i}$ and $\mathrm{j}$ are the coordinates of the blocks, while $\mathrm{x}$ and $\mathrm{y}$ are the pixel coordinates of the input image. $I_{\text {cur }}$ and $I_{\text {pre }}$ are the intensity of the current image and the previous image, respectively.

$$
\begin{gathered}
B M_{a}(i, j)=\frac{1}{16} \sum_{y=4 i}^{4 i+3} \sum_{x=4 j}^{4 j+3}\left|I_{\text {cur }}(x, y)-I_{\text {pre }}(x, y)\right| \\
B M_{b}(i, j)=\frac{1}{9 \cdot 16} \sum_{y=4(i-1)}^{4(i+1)} \sum_{x=4(j-1)}^{4(j+1)}\left|I_{c u r}(x, y)-I_{\text {pre }}(x, y)\right|
\end{gathered}
$$

The amount of texture change in the candidate blocks is calculated using Eq. 9, and the average texture change for a specific interval is calculated using Eq. 10. In the cases in which the amount of texture change is smaller in the candidate blocks than in the expanded blocks, i.e., the mean absolute difference of the candidate blocks is smaller than that of the expanded blocks, the right-hand side of the equation becomes negative, and thus it is substituted by zero. Here, $i$ and $j$ are the coordinates of the candidate blocks, $n$ is the sequential number of texture change, and $\mathrm{N}$ is set to six for low-pass filtering during about one-second duration.

$$
\begin{aligned}
& \operatorname{BV}(\mathrm{i}, \mathrm{j})=B M_{a}(\mathrm{i}, \mathrm{j})-B M_{b}(i, j) \\
& \operatorname{MBV}(\mathrm{i}, \mathrm{j})=\frac{1}{N} \sum_{n=1}^{N} B V(i, j, n)
\end{aligned}
$$




\subsection{Preliminary Decision Adaptive to Global Errors in Input Images}

The amount of average texture change in flame candidate blocks for a specific interval represents the spread and motion of flame. When the figure exceeds a certain reference value, the concerned region is preliminarily determined as a potential fire region, and data accumulated over time will be used for final fire decision. When this approach is applied to actual systems, however, the amount of texture change can be affected by the global error elements within input images that are caused by camera shaking arising from wind or the vibration of instruments. This may lead to a higher possibility of false detection. To overcome this problem, preliminary fire thresholds adaptive to the motion pixel ratio of input images are applied.

The adaptive preliminary fire threshold $T H_{p d}$ is defined as follows. A difference image between the current image and the previous image is created, and the motion pixel ratio is estimated using $\mathrm{N}$, the number of motion pixels; accordingly, the adaptive threshold is determined. Here, $\mathrm{X}$ and $\mathrm{Y}$ are the horizontal and vertical dimensions of the image, and motion pixels are defined as those that exhibit an intensity value difference of three or more. The adaptive preliminary fire threshold is defined as shown in Eq. 11, and $\mathrm{K}$ is experimentally optimized to be five. It may vary between one and five. That reflects the amount of camera shaking caused by wind. As the K value decreases five to one, false alarm increases.

$$
T H_{p d}=K\left(\frac{N}{Y \cdot X}\right)
$$

The preliminary fire decision value is determined based on the adaptive preliminary fire threshold, as shown in Eq. 12. Here, i and $\mathrm{j}$ are the coordinates of the candidate blocks. The resultant figures accumulated over a specific period are used for the final fire decision.

$$
F I R E_{\text {pre }}(i, j)=\left\{\begin{array}{l}
1: M B V(i, j)>T H_{p d} \\
0: \text { otherwise }
\end{array}\right.
$$

\subsection{Final Fire Decision}

In the final fire decision phase, the degree of correlation, average intensity, and preliminary decision value of flame candidate blocks are averaged for a given interval. When it is verified that the final decision conditions have been met, the concerned flame candidate block is confirmed as a fire. Here, the final decision conditions reflect flame and non-flame characteristics and phenomena, as follows. The characteristics of flame and artificial heat-source objects as false detection elements are compared and summarized in Table $\mathbf{1}$.

Table 1. Comparison of characteristics of flame and non-flame objects in thermal images

\begin{tabular}{|c|c|c|ll|}
\hline Division & $\begin{array}{c}\text { Degree of texture } \\
\text { correlation }\end{array}$ & $\begin{array}{c}\text { Average } \\
\text { intensity }\end{array}$ & \multicolumn{1}{|c|}{ Examples } \\
\hline Flame & Low & High & Flame \\
\hline $\begin{array}{c}\text { Dynamic heat-source } \\
\text { objects (non-flame) }\end{array}$ & Low & Low & $\begin{array}{l}\text { Moving vehicles, excavators in } \\
\text { operation, etc. }\end{array}$ \\
\hline $\begin{array}{c}\text { Static heat-source } \\
\text { objects (non-flame) }\end{array}$ & High & High & $\begin{array}{l}\text { Chimneys, streetlights, heating } \\
\text { apparatuses, and sunlight reflection } \\
\text { (rocks, building walls, roofs, etc.) }\end{array}$ \\
\hline
\end{tabular}


Flame and non-flame objects are characterized as follows. Dynamic heat-source objects, such as moving vehicles, move faster than flame can spread, and thus the intensity of the corresponding candidate blocks sharply decreases over time. Also, temporal correlation is low due to position change. Meanwhile, static heat-source objects, such as heating apparatuses or rocks, buildings, and plant chimneys that may reflect sunlight, exhibit a high intensity and thus have a high chance of being selected as candidate blocks; however, these objects are immobile, and thus the high intensity is maintained over time, and the temporal correlation is found to be high because there is no position change. In contrast, flame candidate blocks exhibit a low degree of temporal correlation, while the intensity is maintained at high over time due to the motion and spread of flame, resulting in position change. In consideration of these characteristics, the degree of correlation, intensity, and preliminary decision value of candidate blocks are averaged for a given interval, and the results are used for final fire decision.

$$
\begin{aligned}
& F I R E_{\text {pcnt }}(i, j)=\sum_{m=1}^{M} \operatorname{FIRE}_{\text {pre }}(i, j, m) \\
& B M_{\text {avg }}(i, j)=\frac{1}{N} \sum_{n=1}^{N} B M_{a}(i, j, n) \\
& B I_{\text {avg }}(i, j)=\frac{1}{N} \sum_{n=1}^{N} B I_{b l k}(i, j, n)
\end{aligned}
$$

Here, $\mathrm{i}$ and $\mathrm{j}$ are the coordinates of the blocks, while $B M_{\text {avg }}$ and $B I_{\text {avg }}$ are the degree of correlation and intensity averaged for a given interval, respectively. The number of elements within a given interval, $\mathrm{N}$, was set to 21 , which corresponds to the time taken to conduct three preliminary decisions. $B M_{a}$ is the mean absolute difference of the blocks, and $B I_{b l k}$ is the average intensity of the blocks. FIRE $E_{p c n t}$ is the sum of the preliminary fire decision values for a given interval, and $\mathrm{M}$ was set to 10 . Based on the combination of the three elements described above, a conditional expression for the final decision was formulated, as shown in Eq. 16.

$$
\left(F I R E_{p c n t} \geq 2\right) \&\left(B M_{a v g}>T H_{p d}\right) \&\left(B I_{a v g} \geq T H_{\text {hot }}\right)
$$

\section{Computer Simulation and Results}

To verify the function and performance of the proposed thermal imaging fire detection algorithm, a computer simulation was conducted on ten test videos. Each video used in the simulation is a YCbCr color model-based thermal image recorded at 30 frames per second with a resolution of $720 \times 480$. A total of ten test videos are summarized in Table 2. Among them, seven videos contain actual flame images, while three contain false detection elements, such as sunlight reflection, artificial lighting, vehicles, and excavators. The average recording time is 30 seconds. The sampling interval for testing the proposed algorithm was set to 0.2 seconds. The computer simulation results are presented in Fig. 5. Here, regions confirmed as fire are marked with red circles. 
Table 2. Type and properties of test videos

\begin{tabular}{|c|l|}
\hline No. & Properties of test videos \\
\hline Video $1^{*}$ & Video of a fire from a short distance that contains objects reflecting sunlight (Fig. 5-a) \\
\hline Video $2^{*}$ & $\begin{array}{l}\text { Video of a fire from a far distance that contains screen shaking and multiple objects } \\
\text { reflecting sunlight (Fig. 5-b) }\end{array}$ \\
\hline Video $3^{*}$ & $\begin{array}{l}\text { Video of a fire from a far distance that contains a forest fire with screen shaking (Fig. } \\
\text { 5-c) }\end{array}$ \\
\hline Video 4 & Video that contains a building reflecting sunlight (Fig. 5-d) \\
\hline Video 5 & Video that contains the headlights of a moving vehicle in tunnel (Fig. 5-e) \\
\hline Video 6* & Video from a short distance that contains lighting and torches in tunnel (Fig. 5-f) \\
\hline Video 7* & Video of a fire between buildings from a far distance (Fig. 5-g) \\
\hline Video 8* & Video of a fire on the plain from a far distance (Fig. 5-h) \\
\hline Video 9 & Video contains an excavator in operation (Fig. 5-i) \\
\hline Video 10* & Video of a fire in a town from a short distance (Fig. 5-j) \\
\hline
\end{tabular}

(* video that contains fire scenes)

Fire detection performance measured for each test video is summarized in Table 3. First, a preliminary fire decision was conducted three times on input images fed at an interval of 0.2 seconds. Following that, each input image was processed, and decided with the final decision conditions.

Table 3. Performance test results of proposed algorithm

\begin{tabular}{|c|c|c|c|c|}
\hline Video No. & $\begin{array}{c}\text { No. of fire } \\
\text { decision } \\
\text { (every } 0.2 \mathrm{~s} \text { ) }\end{array}$ & $\begin{array}{c}\text { No. of fire decision } \\
\text { on image with } \\
\text { flames }\end{array}$ & $\begin{array}{l}\text { No. of fire } \\
\text { alarm }\end{array}$ & $\begin{array}{c}\text { No. of false } \\
\text { alarm }\end{array}$ \\
\hline Video $1 *$ & 123 & 123 & 123 & 0 \\
\hline Video $2 *$ & 123 & 123 & 116 & 0 \\
\hline Video 3* & 123 & 123 & 103 & 0 \\
\hline Video 4 & 123 & 0 & 0 & 0 \\
\hline Video 5 & 123 & 0 & 0 & 0 \\
\hline Video 6* & 123 & 123 & 123 & 0 \\
\hline Video 7* & 123 & 60 & 43 & 0 \\
\hline Video 8* & 123 & 123 & 121 & 0 \\
\hline Video 9 & 123 & 0 & 0 & 0 \\
\hline Video $10^{*}$ & 123 & 123 & 123 & 0 \\
\hline Total & 1230 & 798 & 752 & 0 \\
\hline \multicolumn{3}{|c|}{ Total Correct \& False Decision Ratio (\%) } & 94.24 & 0 \\
\hline
\end{tabular}

(* video that contains fire scenes)

The computer simulation results of the test videos showed that the overall decision accuracy of the proposed algorithm was $94.24 \%$ fire alarm ratio, without false alarms. The correct fire decision rate was calculated using Eq. 17.

$$
\frac{\text { No. of fire alarm }}{\text { No. of fire decision with flame }} \times 100(\%)
$$






(a)

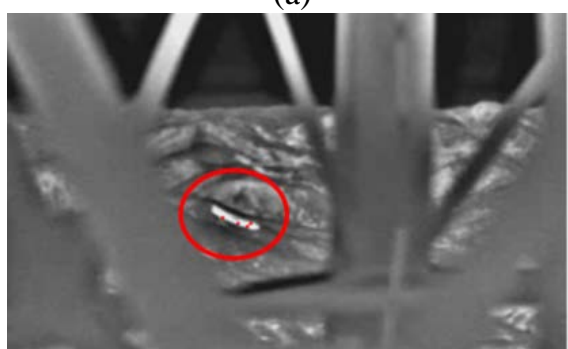

(c)

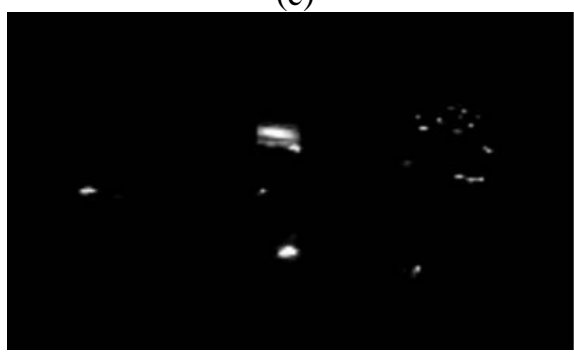

(e)

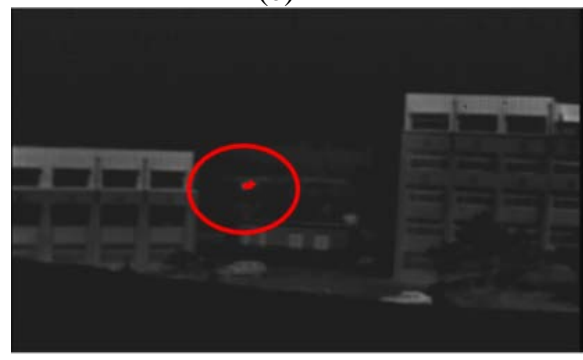

(g)



(i)

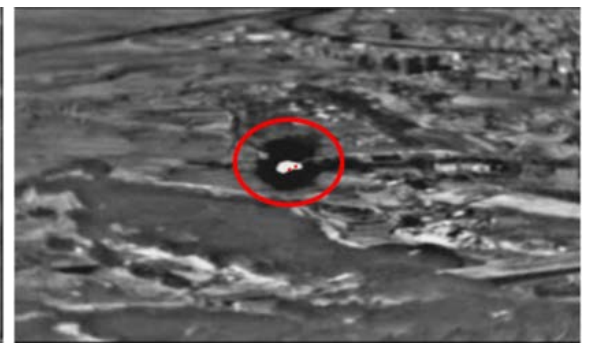

(b)

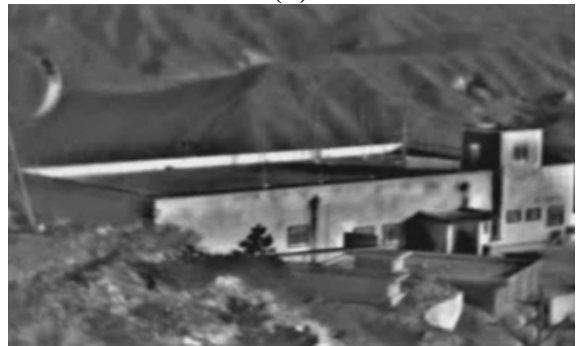

(d)

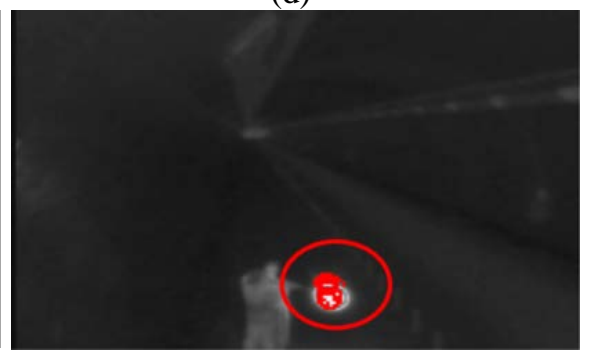

(f)

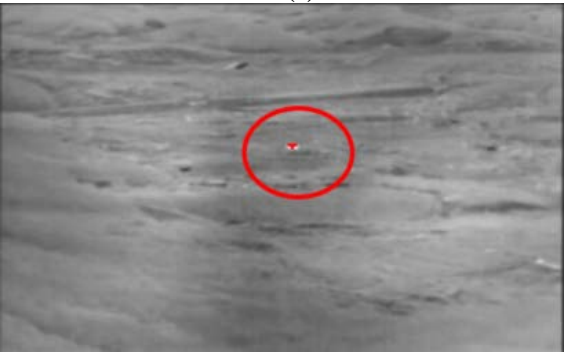

(h)

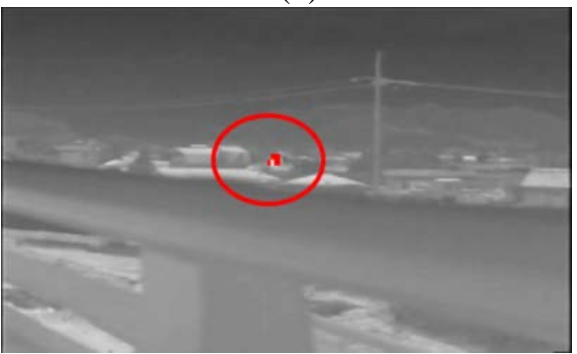

(j)

Fig. 5. Computer simulation results 
As described in Table 2, the seven test videos (video 1,2,3,6,7,8,10) have fire scenes and scenes causing false alarms. And three videos (video 4,5,9) have scenes causing false alarms without fire scenes. Video 1 has fire and rocks reflecting sunlight. In videos 2 and 3, there are fire, camera shaking and objects reflecting sunlight. Video 4 has building reflecting sunlight. Objects reflecting sunlight, and camera shaking may cause false fire detection. Video 5 has headlights of moving cars in tunnel. High-brightness moving headlights may cause false fire detection. In video 6, it contains lighting and person shaking torches in tunnel. High-brightness lighting and moving person may cause false fire detection. In vedeos 7, 8 and 10, there are fire and high-brightness backgound or building. Video 9 has moving excavator. High temperture moving engine may cause false fire detection.

As a result of the simulation, the seven fire videos (video 1,2,3,6,7,8,10) generated correctly fire alarms without false alarms, and three non-fire videos (video 4,5,9) did not generated fire alarms and false alarms. In videos 2 and 3, the camera shaking, which resembled the dynamic characteristics of flame, was likely to cause false detection, but the application of the preliminary threshold adaptive to the global error element caused by the camera shaking ruled out the possibility of false detection. In videos 5 and 9, the vehicles and the excavator were selected as flame candidate regions because of their high engine heat, but the vehicles were ruled out due to the low average intensity. In the case of the excavator in operation, its engine moved in a way similar to that of flame, but the difference in the center of gravity between the heat-source region and hot-source region was significant. In this way, false detection could be avoided. In videos 1, 3, and 4, sunlight-reflecting objects, such as rocks, houses, and artificial structures, were selected as candidate blocks because their intensity levels were very high, but the temporal correlation analysis successfully ruled out the possibility of false detection.

Compared with the performance of the previous algorithm [1], The previous algorithm was found to be inferior in terms of false detection performance, one of the key performance objectives of the proposed algorithm. The previous algorithm resulted in some false detections in Test Videos 2, 3, and 9, while the proposed algorithm demonstrated improved detection performance with zero false detection rate. In the previous algorithm, fire decision was carried out solely based on the fixed threshold-based candidate block selection and the average degree of correlation measured from input images fed at an interval of 0.5 seconds and focused in real-time early detection at video sequence level. This algorithm was mounted on an image processing board, and its video sequence level detection and false detection performance were evaluated at an interval of 3 seconds. The very high fire alarm ratio was checked in video sequence level, but some false alarms were occurred, in addition sometimes correct and false alarms simutaneously occurred in the same image. In the field system, false alarms are very severe problem to be resolved.

\section{Conclusion}

In the present study, a thermal imaging fire detection algorithm was proposed that was designed to be adaptive to the properties of input images and so minimize the false detection rate. First, adaptive flame thresholds were determined based on the average intensity, standard deviation, and maximum intensity of input images, and the centers of gravity of the heat-source and hot-source regions were compared. By doing so, non-flame elements in the early stage could be effectively ruled out. Following that, during the preliminary decision based on correlation analysis, in which texture change of flame candidate blocks in adjacent images was quantified, the thresholds were adaptively set to the motion pixel ratio of input 
images to remove false detection elements caused by global errors, such as camera shaking. In the final fire decision phase, the cumulative preliminary decision values, average intensity change, and average degree of correlation of the flame candidate blocks for a given interval were assessed. When these three decision conditions were met, the concerned candidate block was confirmed as a fire. To verify the performance of the proposed algorithm, seven videos that contained both flame and non-flame elements and three videos that contained only non-flame elements were subjected to computer simulation. The computer simulation results showed that the fire detection accuracy of the proposed algorithm was $94.24 \%$, without false detection, indicating its conformity with the target performance. Also, the proposed algorithm completely ruled out the possibility of false detection, demonstrating significantly improved detection performance and practicality compared to the existing algorithm. A future study will focus on converting the algorithm into one that can be embedded in an image processing board and on implementing it while verifying its real-time processing and detection performance.

\section{References}

[1] Won-Ho Kim, "DSP Embedded Early Fire Detection Method Using IR Thermal Video," KSII Transactions on Internet and Information Systems, vol. 8, no. 10, pp. 3475-3489, 2014. Article (CrossRef Link)

[2] TJokorda Agung Budi W., Iping Supriana Suwardi, "Fire alarm system based on video processing," in Proc. of International Conference on Electrical Engineering Informatics, pp. 1-7, 2011.

Article (CrossRef Link)

[3] Turgay celik, "Fast and efficient method for fire detection using image processing," ETRI journal, vol. 32, no. 6, pp. 881-890, 2010. Article (CrossRef Link)

[4] Suzilawati Mohd Razmi, Nordin Saad, Vijanth Sagayan Asirvadam, "Vision-based flame detection: motion detection \& fire analysis," in Proc. of IEEE Student Conference on Research and Development, pp. 187-191, 2010. Article (CrossRef Link)

[5] Mei Zhibin, Yu Chunyu, Zhang Xi, "Machine vision based flame detection using multi-features," in Proc. of 24 ${ }^{\text {th }}$ Chinese Control and Decision Conference, pp. 2844-2848, 2012.

Article (CrossRef Link)

[6] Zhengwen Xie, Qiang Wang, "Large space fire detection in laboratory-scale based on color image segmentation," in Proc. of ninth International Symposium on Distributed Computing and Applications to Business Engineering and Science, pp. 572-575, 2010. Article (CrossRef Link)

[7] Wirth M., Zaremba R, "Flame region detection based on histogram back-projection," in Proc. of Canadian Conference on Computer and Robot Vision, pp. 167-174, 2010. Article (CrossRef Link)

[8] S.Briz, A.J.de Castro, J.M. Aranda, J. Melendz, F. Lopez, "Reduction of false alarm rate in automatic forest flame infrared surveillance systems," Remote Sensing of Environment, Elsevier Science Inc., vol. 86, no. 1, pp. 19-26, 2003. Article (CrossRef Link)

[9] A.Ollero, B.C.Arrue, J.R.Martinez, J.J. Murillo, “Techniques for reducing false alarms in infrared forest-flame automatic detection systems,” Control Engineering Practice, vol. 7, pp. 123-131, 1999. Article (CrossRef Link)

[10] B.C. Arrue, A. Ollero, J.M de Dios, “An intelligent system for false alarm reduction in infrared forest flame detection,” IEEE Intelligent Systems and their Applications, vol. 15, no. 3, pp. 64-73, 2000. Article (CrossRef Link)

[11] Ignacio Bosch et al, "Infrared image processing and its application to forest fire surveillance," in Proc. of IEEE Conference on Advanced Video and Signal Based Surveillance, pp. 283-288, 2007. Article (CrossRef Link)

[12] W. Philips, M.shah, N.V. Lobo, “Flame recognition in video," in Proc. of the 5th IEEE workshop on application of computer vision, pp. 224-229, 2000. Article (CrossRef Link) 
[13] J.R. Martinez-de Dios et al, “Computer vision techniques for forest flame perception,” Image and Vision Computing, vol. 26, pp. 550-562, 2008. Article (CrossRef Link)

[14] Rafael C. Gonzalez, Digital image processing using MATLAB, Prentice Hall, 2004.

[15] Kenneth ayala. Intel microcontroller. Thomson Delmar learning, 2005.

[16] Nurul Shakira Bakri, Ramli Adnan, Abd Manan Samad, Fazlina Ahmat Ruslan, “A methodology for fire detection using color pixel classification," in Proc. of IEEE International Colloquium on Signal Processing \& its Applications, pp.94-98, 2018. Article (CrossRef Link)

[17] Chongshuang Qin, Chuanliang Guan, Minglun Zhang, Wenfei Sun, Wen He, Hongyu Zhou, “A new real-time fire detection method based on infrared image,” in Proc. of IEEE International Conference on Computer Science and Network Technology, pp.476-479, 2019. Article (CrossRef Link)

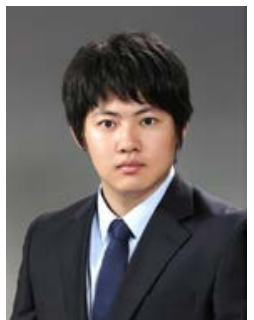

Soo Young Jeong received the B.S. and M.S. degrees in electronics engineering from Kongju National University, Cheonan, Korea, in 2013, 2015, respectively. In 2015, he joined Comesta, Inc. where he is now a senior researcher. His research interests are areas of digital signal processing, image processing, video surveillance system.

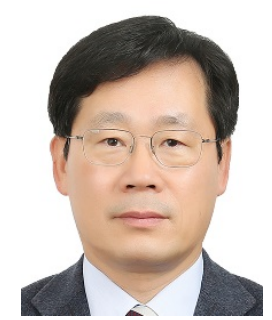

Won-Ho Kim received the B.S. and M.S. degrees in electronics engineering from Kyung-Pook National University, Daegu, Korea, in 1985, 1987, respectively. He received Ph.D. degree in electronics engineering from Chung-Nam National University, Daejeon, Korea, in 1999. From 1989 to 1999, he was with Electronics and Telecommunications Research Institute (ETRI), Daejeon, Korea, as a senior researcher. In 1999, he joined Kongju National University where he is now a full professor. His research interests are areas of digital signal processing, image processing, video surveillance system, and multimedia satellite communication system. 